Erlanger (7), in dem Sphygmoskop von Pal (8) und dem neuen Instrument nach v. Recklinghausen (9) verwertet. v. Recklinghausen nennt diese Methode die oszillatorische.

Das Sphygmoskop, mit dem ich meine Untersuchungen ausgeführt habe, wurde von $\mathrm{Pal}$ in dem Bestreben konstruiert, einen besonders empfindlichen Registrierapparat für die Druckschwankungen in den Arterien zu gewinnen.

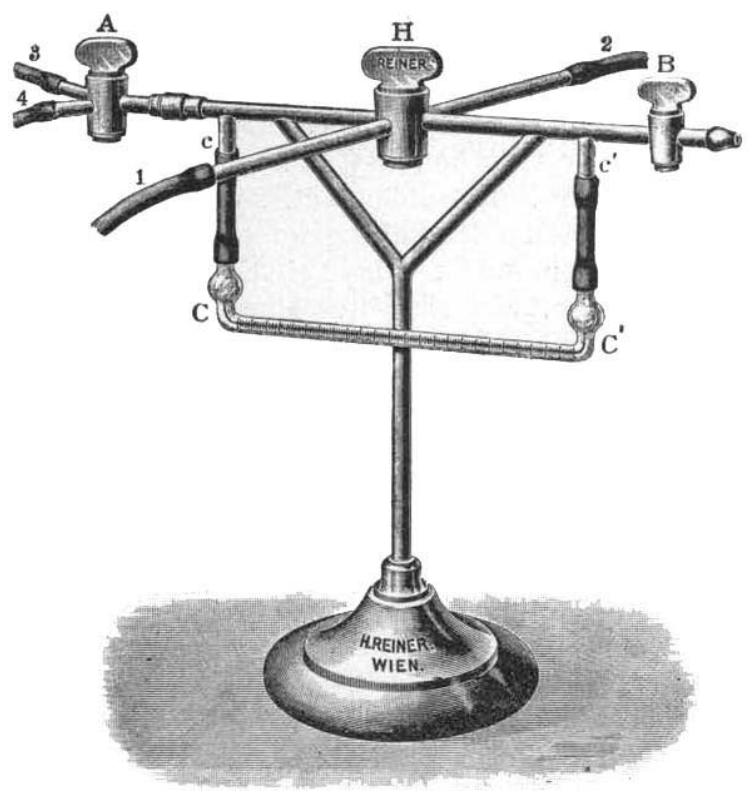

Sphygmoskop nach $\mathrm{P}$ al. $\mathrm{H}=$ Haupthahn, $\mathrm{A}=$ Einstellungshalın für Arm und Finger, $\mathrm{B}=$ Lïftungshahı, $\mathrm{CC}^{1}=$ Capillare.

Die Druckschwankungen werden hier aus einer das GefäBrohr komprimierenden Manschette auf eine capillare Flüssigkeitssäule übertragen, die unter gewissen Bedingungen dadurch in schwingende Bewegung versetzt wird. Als Flüssigkeit wird ein mit Alkanin rotgefärbter Petroleumtropfen verwendet. Die Empfindlichkeit dieses Indikators wird nun dadurch bedeutend erhöht, daß das Prinzip einer "entlasteten Membran“ nach v. Bernd (10) hier auf eine Flüssigkeitssäule übertragen wird. Diese Anordnung hat den folgenden Zweck: Solange ich den Druck in der Manschette steigere, steht der Flüssigkeitstropfen an beiden Enden unter gleichem Druck, befindet sich daher in Ruhe. Sobald der Haupthahn (H) des Sphygmoskops jedoch geschlossen wird, wird zugleich hinter dem Indikator ein Luftraum abgesperrt; dieser wirkt nun gewissermaßen als eine sehr weiche Feder, gegen die die Flïssigkeitssäule bei jeder Druckerhöhung gepreßt wird.

Die Druckbestimmung selbst vollzieht sich in folgender'

Aus der I. Medizinischen Abteilung des k. k. Allgemeinen Krankenhauses in Wien. (Vorstand: Prof. Dr. Pal.)

\section{Ueber Blutdruckuntersuchung mit dem Sphygmoskop nach Pal.}

Voll Dr. Arthur Horner, Assistent der Abteilung.

Die Bestimmung des Blutdrucks beim Menschen erfolgt gewöhnlich in der Weise, daß man die Arterie, deren Seitenwanddruck man messen will, komprimiert. Diese Kompression wird jetzt in der Regel mit einer lufthaltigen Manschette oder einem Luftpolster ausgeführt. Die weitere Ausnutzung dieses Prinzips kann nun auf zweierlei Weise erfolgen. Entweder man beobachtet die Schwankungen, welche die Arterie auf die Manschette überträgt, indem man die letztere mit einer Registriervorrichtung verbindet, oder aber man untersucht den Effekt der allıählichen Kompression an einer peripherwärts gelegenen Stelle der Arterie; dieser Effekt kann palpatorisch (Strasburger [1]) oder sphyginographisch (Janeway [2], Masing [3], Sahli [4]) verfolgt werden. Die Methode, nach welcher die Druckschwankungen in der Manschette beobachtet werden, wurde ursprünglich von Marey (5) angegeben und erscheint u. a. in den Apparaten von Hill und Barnard (6),

1) Pawlowski, Deutsche medizinische Wochenschrift 1892, No. 28. - 2) Holst Ref. Frt. haemat. 1904. - 3) Jousset, Archiv de méd. expérin. et d'anat. patholog. 1905, Bd. 17.
Weise: Wird der Druck in der Manschette ein wenig gesteigert und die Registriervorrichtung durch Schließen des Haupthahus $(\mathrm{H})$ eingeschaltet, so verzeichnet sie in dieser Phase ganz kleine Ausschläge. Wenn wir nun den Druck in der Manschette allmählich noch weiter erhöhen, so gelangen wir zu einem Moment, in dem die Größe der Oszillationen bedeutend zunimmt. Es ist die untere Grenze des Pulsdrucks, der diastolische oder Minimaldruck erreicht, dessen Größe wir an einem mit dem Apparat in Verbindung stehenden Hg-Manometer ablesen können. Eine weitere Steigerung des Manschettendrucks bewirkt zunächst eine fortschreitende Größenzunahme der Oszillationen, die schließlich ihr Maximum erreichen. Diese größten Oszillationen nehmen mit fortgesetzter Kompression des GefäBrohrs wiederum ab, um bei einer bestimmten Druckhöhe ganz kleinen Schwingungen Platz zu machen, die durch das Anschlagen der Pulswelle an den oberen Rand der Manschette bedingt werden. Es ist jetzt die Phase eingetreten, in der das Arterienrohr auch während der Systole verschlossen bleibt, und damit zugleich die obere Grenze des Pulsdrucks, der systolische oder Maximaldruck gegeben. Die Bestimmung der Endwerte kann auch in umgekehrter Richtung ausgeführt werden, indem nämlich zuerst Ueberdruck in der Manschette erzeugt und das Gefäßrohr dann allmählich entlastet wird. Die Wiederholung der Messung in dieser Weise erscheint zugleich als ein geeignetes Verfahren, um die Richtigkeit der zuerst gefundenen Werte zu 
kontrollieren. Bei kleinen Differenzen empfiehlt es sich, aus den so gewonnenen Größen das arithmetische Mittel zu wählen. In derselben Weise wie am Oberarm kann mit diesem Apparat die Druckbestimmung sofort auch an einem Fingergliede, resp. an einem andern Extremitätenabschnitte vorgenommen werden. Die Beurteilung der Flüssigkeitsschwingungen erfolgt in Abständen von $5 \mathrm{zu} 5 \mathrm{~mm}$.

Es entsteht nun die Frage, in welchem MaBe das Sphygmoskop den Anforderungen entspricht, die an ein derartiges Instrument gestellt werden müssen. In dieser Hinsicht sind folgende wichtige Sätze zu beachten, die auch von Oliver aufgestellt wurden: Das Instrument soll genaue Ablesungen des systolischen und diastolischen Drucks ermöglichen. Was diesen Punkt anbelangt, so sei darauf verwiesen, daß die Größenzunahme der Flüssigkeitsoszillationen, bzw. deren Abnahme ganz bedeutend ist, sobald die Endwerte des Pulsdrucks erreicht sind. Es erklärt sich dies einerseits aus der Leichtigkeit des schwingenden Flüssigkeitstropfens, anderseits aus der Empfindlichkeit des Instruments. Zugleich mit der Größenzunahme der Ausschläge ändert sich aber auch deren Charakter, indem die bis dahin kleinen und trägen Schwingungen einen mehr schnellenden, zuckenden Verlauf nehmen. Man gewinnt den Eindruck, als ob erst in dieser Phase die komprimierende Manschette sich den Druckschwankungen im Arterienrohr vollständig anpassen würde. Auch dieser Umschlag ist sehr augenfällig und erhöht die Exaktheit der Bestimmung.

Das Instrument soll ferner genügend empfindlich sein, um von verschieden zugängigen Stellen der peripheren Arterien genaue Werte $\mathrm{zu}$ verschaffen. Diese Forderung wird in erster Linie dadurch erfüllt, daß beim Sphygmoskop, wie bereits erwähnt, das Prinzip einer „entlasteten Membran“ zur Anwendung gelangt; dadurch werden einerseits die Ausschläge des Indikators vergröbert, anderseits wird derselbe befähigt, auch sehr geringe Druckänderungen in der Arterie deutlich zur Anschauung $\mathrm{zu}$ bringen. Bei der Leichtigkeit des schwingenden Tropfens erscheint das Moment der Trägheit so gut wie ausgeschaltet. Eine Feder gelangt in der Apparatanordnung überhaupt nicht zur Verwendung. Die hohe Empfindlichkeit des Instruments ermöglicht auch an den feinsten Verzweigungen der Brachialarterie, in denen die Intensität der Druckschwankungen im Vergleich zum Hauptrohr sehr gering ist, eine exakte Bestimmung des Pulsdrucks. Dabei kann der Apparat schon durch die Umstellung eines Hahnes (A) für Druckbestimmung an einem Fingergliede eingestellt werden. Nur in sehr wenigen Fällen waren die Druckschwankungen in der Art. digitalis so gering, daß sie sich der Beurteilung durch das Sphygmoskop entzogen. Ein Vergleich mit dem von Oliver (11) angegebenen Instrumente lehrte, dab hier in den meisten Fällen von der Digitalarterie aus nur dann Ausschläge sichtbar gemacht werden konnten, wenn jenseits der Flüssigkeitssäule ein entsprechend großer Druckraum eingeschaltet wurde. Gerade aber die Bestimmung des Pulsdrucks an der Art. digitalis erscheint mir von großer Bedeutung. Durch sie wird unsere Einsicht in das Wesen der Kreislaufverhältnisse vermehrt und die Möglichkeit zu weitergehenden Schlüssen auf den Ablauf der Druckschwankungen gegeben.

Es soll ferner die Manipulation des Instrumentes leicht und die Technik keine schwierige sein. Zu diesem Punkte sei bemerkt, daß nach Anlegung der Manschette und Herstellung des gewünschten Druckes die einfache Umstellung eines Hahnes $(\mathrm{H})$ genügt, um die Oszillationen zur Anschauung zu bringen, wozu keine besondere technische Vorbildung notwendig ist. Gerade das letztere Moment sichert unserer Methode ihren Vorzug vor der palpatorischen. Selbst bei guter Ausbildung des Tastsinns, über die nicht jeder Untersucher in gleicher Weise verfügt, ist eine lange fortgesetzte Uebung erforderlich, um die feineren Druckänderungen an der Radialarterie wahrnehmen zu lernen. Dazu kommt noch die Ermüdung des Tastsinns, die bei längeren Untersuchungen ihren störenden Einflub geltend macht. Das Verfahren ist sonach ein allzu subjektives, während die optische Verfolgung der Flüssigkeitsschwingungen ein relativ objektives Urteil ermöglicht, um so mehr, als sie von mehreren Beobachtern gleichzeitig durchgeführt werden kann. Ein weiterer Nachteil der palpatorischen Methode liegt darin, daß sie nur für den Oberarm anwendbar ist, somit nur an einem beschränkten Stromgebiet der Arterienbahn Druckbestimmungen gestattet.

Vor dem neuen Apparate nach v. Recklinghausen $\left.(9)^{1}\right)$ hat das Sphygmoskop den Vorzug, daß darin keine Feder zur Verwendung gelangt, daß die Druckablesung direkt an dem Hg-Manometer erfolgt, die Leichtigkeit der Einstellung für Oberarm und Finger und schließlich die größere Amplitude der Oszillationen, die die exakte Fixierung der Grenzwerte begünstigt. Von dem Apparate nach Erlanger (7), der das Resultat der Druckinessung graphisch darstellt, unterscheidet sich das Sphygmoskop vorteilhaft durch die wesentlich einfachere Beschaffenheit und Manipulation, dab sie keiner besonderen Vorbereitungen bedarf und rasch durchführbar ist.

Schwierigkeiten bereitet die Bestimmung des Pulsdrucks in Fällen von inäqualem Puls. Wo diese Ungleichheit der Pulse nur wenig ausgesprochen war, haben wir nur auf den größten Pulsschlag Rücksicht genommen. In Fällen bedeutenter Inäqualität sind wir so vorgegangen, daß wir die Endwerte für den gröbten und kleinsten Pulsschlag bestimmten und aus den entsprechenden Größen das arithmetische Mittel nahmen.

Als Manschette für den Oberarı benutzen wir gegenwärtig die neue Manschette nach v. Recklinghausen (9). Diese unterscheidet sich von der von Sahli angegebenen durch ihre größere Breite $(13 \mathrm{~cm})$. Auch wir konnten die Beobachtung vieler Autoren bestätigen, daß eine schmale Manschette für den systolischen Druck höhere Werte ergibt als eine breitere; eine Differenz, die unter Umständen eine ziemliche Größe erlangen kann. Dies gilt namentlich für jene Fälle, in denen der Oberarm von der zylindrischen Form stärker abweicht. Erlanger (7) hat die Frage nach der Größe dieses Fehlers, der sich aus der Wahl einer zu schmalen Manschette ergibt, experimentell studiert und gefunden, dab dieser nit der Breite der Manschette abnimmt. Für die Druckbestimmung an einem Fingergliede benutzen wir den breiten, weichen Fingerring nach Gärtner. Der im System gewünschte Druck kann durch einen Ballon mit Kompressorium reguliert werden, seine Ablesung erfolgt an einem Uförmigen Hg-Manometer.

Der Venendruck, bzw. der im rechten Vorhof herrschende Druck wurde in den von uns untersuchten Fällen nach dem Prinzip von Frey (12)-Gärtner (13) gemessen. Es wurde zunächst die Höhe bestimmt, in der beim Heben des Armes ein Kollaps der Vorderarmvenen eintrat, hierauf der Arm langsam gesenkt und der Moment fixiert, in dem sich diese Venen wieder mit Blut füllten. Aus den so gewonnenen Grenzwerten wurde dann das arithmetische Mittel als Ausdruck des im rechten Vorhof herrschenden Drucks gezogen. Diese von mir (14) seinerzeit angegebene Modifikation hat überdies „den Vor: teil, daß Ungenauigkeiten, die bei Beurteilung der Füllungsverhältnisse der Venen kaum zu vermeiden sind, auf die Hälfte reduziert werden". Als Ausgangspunkt dieser Messungen diente der obere Rand der fünften Rippe an ihrem Sternalansatze.

Bevor ich daran gehe, die Leistung des Sphygmoskops an einigen pathologischen Fällen zu demonstrieren, möchte ich zunächst die Messungsresultate an gesunden Individuen kurz mitteilen. Die Bestimmungen wurden an einer großen Zahl erwachsener Personen beiderlei Geschlechts im mittleren Lebensalter ausgeführt und ergaben das folgende Resultat:

Systol. Druck Mittelwert Diastol. Druck Mittelwert Pulsdruck Mittelwert $\begin{array}{lcccccc}\text { Oberarm } & 100-130 & 115 & 50-70 & 60 & 50-60 & 55 \\ \text { Grundphal. } & 85-115 & 100 & 40-60 & 50 & 45-55 & \mathbf{5 0}\end{array}$

Vergleichen wir die Normalzahlen, welche mittels verschiedener Messungsmethoden ermittelt wurden, miteinander, so ergeben sich ziemlich bedeutende Differenzen, sodaß von den vielen Beobachtungen, die nach dieser Richtung hin angestellt wurden, kaum zwei miteinander vollständig übereinstimmen. Dieser Umstand, der immerhin den Wert der Druckmessungen als etwas zweifelhaft erscheinen lassen könnte, dürfte jedoch wenig ins Gewicht fallen, solange bei den verschiedenen Bestimmungen stets nach demselben Prinzip vorgegangen wird.

1) Ich hatte im vergangenen Jahre Gelegenheit, an der Klinik des Geh. Rats Prof. Kra us in Berlin mit diesem Apparate Messungen auszuführen. 
Anderseits darf das Hauptgewicht nicht auf einzelne Messungen, die ja keine absoluten Werte darstellen, gelegt werden, es muß vielmehr der Ablauf der Druckschwankungen, wie er sich aus einer kontinuierlichen Folge von Beobachtungen ergibt, in erster Linie Berücksichtigung erfahren, ein Prinzip, das bei unseren Messungen stets eingehalten wurde. Im allgemeinen haben unsere Bestimmungen einen etwas höheren Pulsdruck ergeben, als er von vielen anderen Autoren gefunden wurde. Die Messung des systolischen Druckes dürfte, nach verschiedenen Methoden vorgenommen, ziemlich übereinstimmende Resultate ergeben. Dagegen scheint mir über die Frage, in welchem Moment der diastolische Druck erreicht ist, nicht vollständige Uebereinstimmung der Anschauungen $\mathrm{zu}$ herrschen. Während von manchen Autoren das Maximum der Oszillationen als untere Grenze des Pulsdrucks angesehen wird, haben wir nach den Ausführungen Pals (8) den Druck an der untersten Grenze großer Ausschläge als Minimaldruck fixiert. Dazu kommt noch, daß bei der hohen Empfindlichkeit des Instruments die Größen- und Formveränderung der Oszillationen und damit das Eintreten des diastolischen Drucks früher in Erscheinung treten dürfte, als dies mit anderen Methoden der Fall ist. Beide Momente würden unsere etwas höheren Zahlen für den Pulsdruck erklären.

In allen unseren Fällen wurden nicht nur am Oberarm, sondern stets auch am ersten Fingergliede, sowie an den Vorderarmvenen Bestimmungen ausgeführt und diese in den Kreis der Betrachtung gezogen. Dieses Verfahren erscheint bis jetzt wenig in der Literatur berücksichtigt, indem sich die meisten Autoren mit der Messung des Pulsdrucks am Oberarm begnügen. Gerade aber die Vergleichung der Druckwerte an möglichst vielen Stellen der Strombahn ist geeignet, unsere Kenntnisse über den Ablauf der Druckschwankungen zu vervollständigen.

Auf dieses Moment hat auch Sihle (15) hingewiesen, der meint, daß „erst durch die Kenntnis der Druckdifferenz zwischen Brachialis und Digitalis eine brauchbare Handhabe zur Beurteilung der Zirkulationswiderstände im untersuchten Gefäßgebiet gegeben sei, während die Untersuchungen an nur einer Stelle über diese Leitungs widerstände sehr wenig aussagen ${ }^{\circ}$. Sihle (15) findet, daß diese Druckdifferenz beim gesunden Menschen etwa $30-40 \mathrm{~mm} \mathrm{Hg}$ betrage und selten den Wert von $50 \mathrm{~mm}$ übersteige. Bei seinen Messungen ist jedoch nur der systolische Druck berücksichtigt, während gerade der diastolische Druck in vielen Fällen geeignet erscheint, über die peripherischen Widerstände Aufschluß zu geben. Olive $r$ (11) findet, daß sich der Blutdruck vom Oberarm bis zum Handgelenk nicht wesentlich ändert und erst am ersten Fingergliede einen Abfall zeigt. Nach seinen Untersuchungen betragen die beiden Grenzwerte für den Pulsdruck beim gesunden Menschen 125 und $100 \mathrm{~mm}$ am Oberarm, 105 und 85 an der Grundphalange. An der zweiten Phalange sinke der systolische Druck auf 80 , der diastolische auf $85 \mathrm{~mm}$. Die beiden Druckwerte nähern sich nach seiner Beobachtung einander desto mehr, je mehr man sich den Endarterien und Arteriolen nähert, wobei der systolische Druck in stärkerem Maße abnimmt als der diastolische.

In den von mir untersuchten normalen Fällen bestätigte eine Vergleichung der am Oberarm und ersten Fingergliede gewonnenen Zahlen die schon von Pal (8) hervorgehobene Erscheinung, daß der Pulsdruck an der Brachialis den entsprechenden Wert an der Digitalis nur um ein Geringes übertreffe. Diese Differenz betrug durchschnittlich $5 \mathrm{~mm}$. Der systolische Druck verringerte sich auf diesem Wege um etwa $15 \mathrm{~mm}$, der diastolische um $10 \mathrm{~mm} \mathrm{Hg}$. Messungen, die gelegentlich am zweiten Fingergliede ausgeführt wurden, ergaben meist keine wesentliche Abweichung von den an der Grundphalange gewonnenen Zahlen. Die Vorderarmvenen entleerten sich in diesen normalen Fällen in einem Niveau, das dem oberen Rande der fünften Rippe an ihrem Sternalansatz entsprach, oder nur um wenige Zentimeter höher gelegen war Neben den Zahlen für den Venenkollaps erscheint in meinen Beobachtungen auch die Höhe angegeben, in der sich die Venen wieder mit Blut füllten sowie der das arithmetische Mittel aus den so gewonnenen Endwerten.

Aus der großen Zahl der bis jetzt beobachteten pathologischen Fälle sollen hier einige herausgegriffen werden, in denen der Verlauf der Druckschwankungen in den verschiedenen Stadien eine Zirkulationsstörung unter der Einwirkung therapeutischer Maßnahmen verfolgt wurde. Von einer systematischen Darstellung der Kreislaufstörungen und ihrem Einflu0 auf die Blutdruckverhältnisse will ich hier absehen. Zunächst bringe ich einige Fälle, die den Effekt der Digitalisdarreichung darstellen. Als Digitalispräparat verwenden wir seit längerer Zeit fast ausschlieblich das Fluidextrakt von Parke, Davis u. Co.

Fall 1. Marie V., 15 Jahre alt. Mitral-Insuffizienz und Stenose.

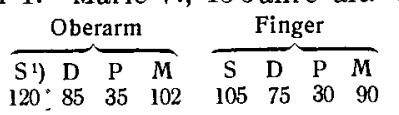

Datum S1)

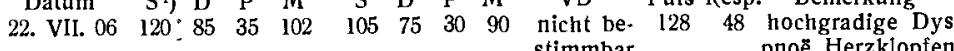

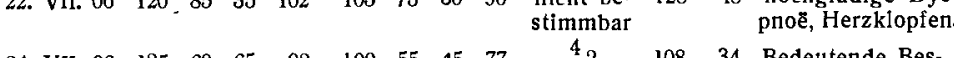
24. VII. $\begin{array}{lllllllllllll}06 & 125 & 60 & 65 & 92 & 100 & 55 & 45 & 77 & { }_{0}^{4} & 108 & 34 & \text { Bedeutende Bes- }\end{array}$ serung nach Extr.

Ein Blick auf die vorliegende Tabelle lehrt zunächst, daß die Zahlen für den systolischen Druck vor der Digitalisdarreichung fast vollständig der Norm entsprechen, trotzdem eine hochgradige Kompensationsstörung subjektiv und objektiv zu verzeichnen war. Hingegen erscheint der diastolische Druck sowohl an der Brachialis als auch an der Digitalis erhöht, der Pulsdruck infolgedessen herabgesetzt; eine Erscheinung, die im Sinne eines Kreislaufhindernisses zu verwerten ist. Der VD war in dieser Phase der Beobachtung nicht meßbar. Unter Digitalisdarreichung besserte sich das Befinden der Patientin. Wir finden bei Betrachtung der Zahlen vom 24. VII., daß auch hier der systolische Druck keine wesentliche Abweichung von der Norm aufweist, die diastolische Druckerhöhung hingegen geschwunden ist, somit die Abflußverhältnisse für das Blut sich gebessert haben. Der VD zeigt keine wesentliche Veränderung. Auffällig bleibt immerhin, auch im Stadium der Kompenration, die stärkere Differenz zwischen dem Pulsdruck an der Brachial- und Digitalarterie, die wir bei vollständig gesundem Herzen gewöhnlich vermissen. In dieser Phase ist es nur der Vergleich der beiden letzteren Größen, der auf das Pathologische des Falles hinweist.

Fall 2. Johann B., 32 Jahre alt. Mitral-Insuffizienz und Stenose.

Datum $\overbrace{\mathrm{S} \text { D P M }}^{\text {Oberarm }}$

17. VII. $06 \begin{array}{lllll}135 & 80 & 55 & 107\end{array}$

i h p. $\mathrm{m}$.

6 h p. m.

$\begin{array}{llll}125 & 55 & 70 & 90\end{array}$

18. VII. $06 \quad 130 \quad 80 \quad 50 \quad 105$

19. VII. $06 \quad 130 \quad 60 \quad 70 \quad 95$

$$
\begin{aligned}
& \text { Finger }
\end{aligned}
$$

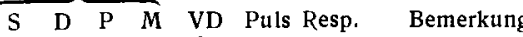

$$
\begin{aligned}
& \begin{array}{lllllllll}
120 & 70 & 50 & 95 & { }_{7}^{13} 10 & 84 & 20 & \text { Dyspno\&, Herzklopfen, }
\end{array}
\end{aligned}
$$

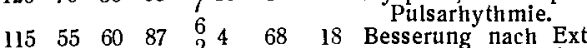

$$
\begin{aligned}
& \begin{array}{l}
\text { 18 Besserung nach Extr } \\
\text { fluid. Digit. } z \text { weima }
\end{array} \\
& \begin{array}{llllllll}
120 & 75 & 45 & 97 & 17 & 13,5 & 86 & 24
\end{array} \quad \text { Zunehmende Be- } \\
& \begin{array}{lllllllll}
120 & 55 & 65 & 87 & { }_{4}^{8} & 78 & 24 & \begin{array}{c}
\text { Besserungerden. } \\
\text { Diuretin. }
\end{array}
\end{array}
\end{aligned}
$$

Fassen wir zunächst die Zahlen vom 17. VII. ins Auge, so konstatieren wir eine ziemlich gleichmäßige Zunahme der Werte für den systolischen und diastolischen Druck gegenüber der Norm. Der Pulsdruck selbst zeigt sowohl am Oberarm als am Finger normale Werte, der VD erscheint wiederum ziemlich stark erhöht. Nach Darreichung von Digitalis ändern sich die Verhältnisse insofern, als die Zahlen für den systolischen Druck eine geringe, die für den diastolischen Druck eine stärkere Abnahme erfahren, der Pulsdruck somit steigt. Der VD zeigt in diesem Stadium fast normale Größe. Alle diese Momente weisen darauf hin, da $\beta$ die Widerstände in der Peripherie abgenommen haben, die Kreislaufverhältnisse günstiger geworden sind. Auch Fellner (16) hat die Beobachtung gemacht da $\beta$ in Fällen von Herzinsuffizienz ein Sinken des Blutdrucks mit Steigen des Pulsdrucks und Verbesserung der Herzaktion einhergeht.

Zu ähnlichen Resultaten führten mich (14) bereits früher meine Untersuchungen über den VD und dessen Beziehungen zu dem arteriellen Druck. Es zeigte sich nämlich in den meisten Fällen von Herzinsuffizienz, daß der arterielle Druck, der dem gesteigerten VD entsprach, keine Herabsetzung, sondern im Gegenteil eine Zunahme aufwies. In der weiteren Beobachtung dieser Fälle konnte ich den Satz aufstellen: „Die eintretende Besserung in den Kreislaufsbedingungen war wiederum mit einer Abnahme des VD, sowie auch des arteriellen Drucks verbunden." Diese Erscheinung suchte ich durch die Annahme zu erklären, daß die Besserung der Zirkulationsbedingungen durch die Aktion des rechten Herzens bedingt sei. Meine damaligen Beobachtungen werden nun durch die vergleichende Messung des diastolischen Drucks vervoll-

1) $\mathrm{S}=$ systolischer Druck, $\mathrm{D}=$ diastolischer Druck, $\mathrm{P}=\mathrm{Puls}$ druck, $M=$ arithmetisches Mittel aus $\mathrm{S}$ und $\mathrm{D}$. Ohne auf die Frage des mittleren Drucks hier näher eingehen zu wollen, sei nur erwähnt daß nach den herrschenden Anschauungen diese Größe näher dem diastolischen als dem systolischen Druck liegt. VD $=$ Druck im rechten Vorhof. 
ständigt. Dieselben zeigen, daß neben der Verbesserung der Herztätigkeit durch therapeutische Maßnahmen auch der Herabsetzung der peripheren Widerstände ein wesentlicher Einflub im Sinne der Kompensation zukommt. Nur möchte ich erwähnen, daß diese Veränderung der Druckverhältnisse nichts Spezifisches für die Digitaliswirkung allein enthält, da dieselbe auch nach Diuretindarreichung und auch anderen therapeutischen Maßnahmen beobachtet werden konnte.

Die weitere Beobachtung des Falles zeigte mit dem Wiederauftreten der bedrohlichen Erscheinungen am 18. VII. ein Ansteigen sämmtlicher Druckwerte. Diese betreffen namentlich den diastolischen Druck sowie den VD, Symptome, die wir bereits als Ausdruck zunehmender Stauung kennen gelernt haben. Eine Besserung der Kreislaufstörung wurde diesmal durch Diuretin bewirkt. Dieselbe gibt sich durch das Sinken des diastolischen Drucks und des VD zu erkennen, der systolische Druck bleibt unverändert. Auffallend ist in diesem Falle immerhin die, wenn auch geringe Zunahme des Pulsdrucks im Stadium der Kompensation.

Fall 3. Franz J., 40 Jahre alt. Mitral-Insuffizienz.

$$
\overbrace{S \quad D \quad P \quad M}^{\text {Oberarm }}
$$

$$
\text { Finger }
$$

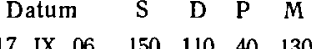

$$
\overbrace{\text { S D P M VD Puls Res }}
$$$$
{ }^{20} \underset{\text { bestimmbar }}{\text { nicht }}{ }^{12} 10 \quad 100 \quad 38 \text { Dyspnoë, Herzklopfen, }
$$

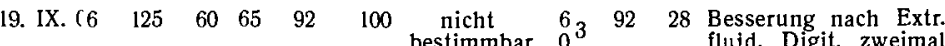$$
10 \text { Tr. tăgl. }
$$

Auch in diesem Falle lassen sich die erwähnten Beziehıngen zwischen Kreislaufstörung und Blutdrucksverhältnissen gut verfolgen.

\begin{tabular}{|c|c|c|c|c|c|c|c|c|c|c|}
\hline \multirow[b]{2}{*}{ Datum } & \multicolumn{4}{|c|}{ Oberarm } & \multicolumn{4}{|c|}{ Finger } & \multirow[b]{2}{*}{ Puls Resp. } & \multirow[b]{2}{*}{ Benıerkung } \\
\hline & S & D & $\mathbf{P}$ & $\vec{M}$ & $\mathrm{~s}$ & D & $P$ & $M$ & & \\
\hline 1. VIII. 06 & 125 & 75 & 50 & 100 & 110 & 65 & 45 & 87 & ${ }_{4}^{10} 7$ & Dyspnoë, Oedem der \\
\hline 8. $1 \mathrm{X} .06$ & 110 & 45 & 65 & 77 & 100 & 40 & 60 & 70 & ${ }_{4}^{8} 6$ & $\begin{array}{l}\text { Wesentliche Besse- } \\
\text { rung nach Diuretin, } \\
3 \mathrm{~g} \text { tăgl. Oedeme ge- } \\
\text { schwunden. }\end{array}$ \\
\hline
\end{tabular}

Fall 4. Johann W., 29 Jahre alt. Mitral-Insuffizienz und Stenose, A.orten-Insuffizienz.

Nach Darreichung von Diuretin finden wir hier dieselben Veränderungen, die in den vorher beschriebenen Fällen die DigitalisMedikation bewirkt hat: Sinken des systolischen und diastolischen Drucks, des letzteren in noch höherem Maße, wodurch der Pulsdruck vermehrt wird, sowie eine, wenn auch sehr geringe Abnahme des VD.

Fall 5. David M., 47 Jahre alt. Arteriosklerose, Stenokardie mit Asthma cardiale,

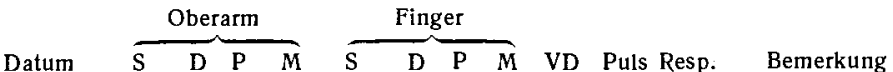

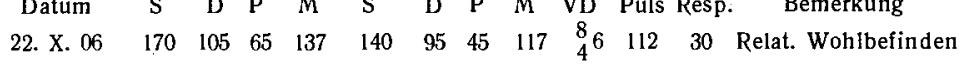

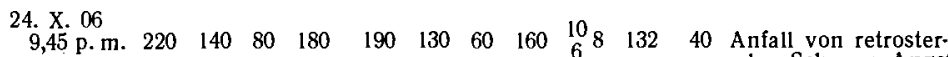

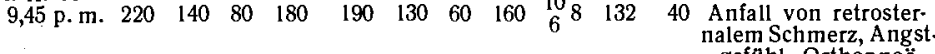
gefahh, Orthopnoe

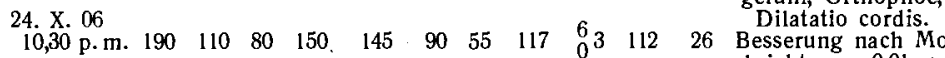
$\begin{array}{llllllllllllll}\text { 26. X. } 06 & 145 & 90 & 55 & 117 & 135 & 85 & 50 & 110 & 0_{3} & 106 & 26 & \begin{array}{c}\text { Injekt. von } 0,01 \mathrm{~g} \\ \text { Wohlbefinden. }\end{array}\end{array}$

In diesem Falle erscheinen die Veränderungen während eines Anfalles von Stenokardie verfolgt. Wir finden auf der Höhe desselben ein Ansteigen sämtlicher Druckwerte als Ausdruck vermehrter Herzarbeit gegen erhöhte Widerstände. Sobald die bedrohlichen Erscheinungen nachlassen, zeigen diese Größen wieder eine Tendenz zur Abnahme. Am 26. X. fühlte sich der Patient wohl. Bemerkenswert erscheint, daß die ziemlich bedeutende Differenz zwischen dem Pulsdruck an der Brachialis und Digitalis, die wir bei der vorhergehenden Messung konstatieren konnten, an diesem Tage geschwunden ist; eine Erscheinung, die wir bereits als ein Symptom verbesserter Zirkulationsverhältnisse kennen gelernt haben.

Nachfolgend will ich noch ganz kurz zwei Fälle anführen, aus denen der Einfluß einer Abdominalpunktion auf die Druckverhält-

\begin{tabular}{|c|c|c|c|c|c|c|c|c|c|c|c|c|}
\hline \multirow{4}{*}{$\begin{array}{l}\text { Datum } \\
\text { 18. IX. } 06 \\
9 \text { h a. m. } \\
\text { 18. IX. } 06 \\
6 \text { h p. m. }\end{array}$} & \multicolumn{4}{|c|}{ Oberarm } & \multicolumn{4}{|c|}{ Finger } & \multirow[b]{2}{*}{ VD } & \multirow[b]{2}{*}{ Puls } & \multirow[b]{2}{*}{ Resp. } & \multirow[b]{2}{*}{ Bemerkung } \\
\hline & S & D & $P$ & $M$ & $\mathrm{~s}$ & D & $\mathbf{P}$ & $\vec{M}$ & & & & \\
\hline & 125 & 80 & 45 & 105 & 105 & 80 & 25 & & & 104 & 48 & Vor der Punktion. \\
\hline & 110 & 50 & 60 & 80 & 105 & 45 & 60 & & & 98 & 36 & $\begin{array}{l}\text { Nach Entleerung von } \\
91 \text { Ascitesflüssigkeit. }\end{array}$ \\
\hline
\end{tabular}
nisse ersichtlich ist:

Fall 6. Helene W., 17 Jahre alt. Polyserositis, Ascites.

Hier sei die Herabsetzung des diastolischen Drucks und die dadurch bedingte Steigerung des Pulsdrucks nach Entlastung der Bauchgefäße hervorgehoben; dadurch wurden wieder ziemlich normale Druckverhältnisse geschaffen.

\begin{tabular}{|c|c|c|c|c|c|c|c|c|c|c|}
\hline \multirow[b]{2}{*}{ Datum } & \multicolumn{4}{|c|}{ Oberarm } & \multicolumn{4}{|c|}{ Finger } & \multirow[b]{2}{*}{ Puls Resp. } & \multirow[b]{2}{*}{ Bemerkung } \\
\hline & $\mathrm{s}$ & $\bar{D}$ & $\mathrm{P}$ & $\vec{M}$ & $\mathrm{~s}$ & D & $\mathrm{P}$ & M & & \\
\hline & 145 & 85 & 601 & 115 & 110 & 60 & 50 & 85 & $106 \quad 36$ & Vor der Punktion. \\
\hline IX. 06 & 120 & 60 & 60 & 90 & 110 & 55 & 55 & 82 & ${ }_{4}^{10} 7$ & $\begin{array}{l}\text { Nach Entleerung von von } \\
\text { 12 I Ascitesflüssigkeit. }\end{array}$ \\
\hline
\end{tabular}

Fall 7. Philippine K., 17 Jahre alt. Mitral-Insuffizienz und Stenose, Tricuspid.-Insuffizienz.
Auch in diesem Falle sinken die vor der Punktion gesteigerten Druckgrößen am Oberarm nach Ausführung des Eingriffs zll normalen Werten herab. Während die Druckmessung an den Arterien nach eingetretener Besserung nichts Auffalliges bietet, weist nur der erhöhte VD auf die schwere Herzaffektion hin.

Diese wenigen Beispiele beweisen schon, daß wir mit Hilfe des Sphygmoskops imstande sind, alle jene Messungen auszuführen, die uns einigen Einblick in das Wesen der Kreislaufstörungen gewähren können. Dieselben liaben uns unter anderem mit zwei neuen Erscheinungen bekannt gemacht, die sich auf das Verhältnis von Pulsdruck am Oberarm und Finger beziehen, und die ich in die folgenden Sätze zusammenfassen möchte

1. Eine größere Differenz zwischen dem Pulsdruck an der Brachial- und Digitalarterie deutet auf das Bestehen einer Kreislaufstörung hin.

2. Diese Differenz verringert sich in manchen Fällen zugleich mit eintretender Besserung der Zirkulationsverhältnisse.

Die Bestimmungen sollten ferner zeigen, wie notwendig es ist, auf möglichst viele Momente bei der Beurteilung unserer Untersuchungsergebnisse Rücksicht zu nehmen, indem in dem veränderlichen Bilde der Blutzilkulation bald die eine, bald die andere Komponente stärkel in Erscheinung tritt. Gerade diese Labilität der Druckverhältnisse bietet eine der Hauptschwierigkeiten in der Lösung der Kreislauffrage. Immerhin aber sind zahlreiche fortgesetzte Beobachtungen bei vorurteilsloser Beurteilung imstande, unsere Kenntnisse auch auf diesem Gebiete zu erweitern. Zum Schlusse möchte ich bemerken, daB ich in meinen Ausführungen bemüht war, nur die rein praktische Seite des Gegenstandes zu erörtern, mich daher von theoretischen Betrachtungen möglichst ferngehalten habe.

Literatur: 1. Strasburger, Ein Verfahren zur Messung des diastolischen Blutdrucks etc. Zeitschr. f. klin. Med. 1904, Bd. 54. - 2. J a n ew a y, Some observations on the estimation of blood pressure in man. N. Y. Bull. of med. sciences 1901, Vol. 1. - 3. Masing, Verhalten des Blutdruckes des jungen und bejahrten Menschen. Deutsches Arch. f. klin. Med. 1902, Bd. 74. - 4. Sa h1 i, Ueber das absolute Sphygmogramm etc. Deutsches Arch. f. klin. Med. 1904, Bd. 51. - 5. Ma re y, Travaux du laboratoire. 1876, T. II. - 6. Hill and B arnard, A simple pocket sphygmanometer for estimating arterial pressure in man. The Journ. of Physiol. Vol XXIII. 1898-1899. - 7. Erl a n ger, A new instrument fo: determining etc. The Johns Hopkins Hosp. Rep. Vol XII 1904 - 8 . P a l. Ein Sphygmoskop zur Bestim. mung mung des Pulsdıucks. Zentralblatt f. innere Med. 1906, No. 5. - 9. v. Re cklingha us en, Unblutige Blutdruckmessung. Arch. f. exper. Pathol. u. Pharmakop. 1906, Bd. 55. - 10. v. B e rnd, Die Verwendung einer "entiasteten Membran“ zur Sphygmographie und Tonographie. Wiener klin. Wochenschr. 1906, No. 2. - 11. O liver, A lecture on haemomanometry in man. Lancet 1905, July 22. - 12 F r e y, Archiv $f$ klin. Med. 1902, Bd. 73. - 13. Gärt ne r, Münch. med. Wochenschr. 1903, No. 47. 14. H orn er, Ueber den Vorhofdruck bei Klappenfehlern. Münch. med. Wochenschr. 1904, No. 32. - 15. Sihle, Beitrăge zu einer funktionellen Diagnose der Arteriosklerose etc. Wien. klin. Wochenschr. 1904, No. 14. -16 . F e $11 \mathrm{n}$ e r, Klinische Beob. achtungen über den Wert der Bestimmung der wahren Blutgrởe etc. Deutsches Arch. f. klin. Med. 1906, Bd. 88 